\title{
Use of glasgow-blatchford bleeding score reduces hospital stay duration and costs for patients with low-risk upper GI bleeding
}

Authors

Institution
Marc Girardin, David Bertolini, Saskia Ditisheim, Jean-Louis Frossard, Emiliano Giostra, Nicolas Goossens, Isabelle Morard, Thai Nguyen-Tang, Laurent Spahr, Alain Vonlaufen, Antoine Hadengue, Jean-Marc Dumonceau

Division of Gastroenterology and Hepatology, Geneva University Hospital, Geneva, Switzerland submitted

3. November 2013 accepted after revision 23. January 2014

\section{Bibliography}

DOI http://dx.doi.org/

10.1055/s-0034-1365542

Published online: 7.5.2014

Endoscopy International Open

2014; 02: E74-E79

(c) Georg Thieme Verlag KG

Stuttgart · New York

E-ISSN 2196-9736

\section{Corresponding author}

Marc Girardin, MD

4 Gabrielle-Perret Gentil Street

1211 Geneva 14

Switzerland

marcgirardin@gmail.com
Background and study aims: Upper gastrointestinal (UGI) bleeding is a frequent cause of hospitalization. Its severity may be assessed before endoscopy using the Glasgow-Blatchford Bleeding Score (GBS), a score validated to identify patients requiring clinical intervention. The aim of this study was to assess whether the GBS was effective for shortening hospital stay and reducing costs in patients with an UGI bleeding predicted at low risk of requiring clinical intervention.

Patients and methods: Consecutive outpatients presenting with UGI bleeding at our hospital were prospectively included. In the observational study phase, UGI endoscopy was performed in all patients according to routine clinical practice. In the interventional study phase, patients with a GBS of 0 were discharged with an appointment for an outpatient UGI endoscopy. All patients had follow-up at 7 and 30 days. Need for clinical intervention was defined as performance of endoscopic hemostasis, blood transfusion or surgery. Results Two-hundred and eight patients were in-

\section{Introduction}

$\nabla$

Upper gastrointestinal (UGI) bleeding is a frequent cause of emergency admission and hospitalization. In the United States, its incidence is 1/ 1000 individuals per year and it accounts for more than 100000 hospital admissions per year at a cost of more than 2 billion USD [1,2]. In the UK, the incidence of non-variceal UGI bleeding was reported to be 50-150/100 000 [3]. In the United States, the costs for variceal and non-variceal UGI bleeding were estimated to vary between 3400 and 23300 USD [4,5].

The severity of UGI bleeding is highly variable; severe episodes account for a small proportion of all bleeding [3]. Several validated scores, based on clinical and endoscopic findings, attempt to predict the rebleeding risk $[6,7]$. UGI bleeding with a low rebleeding risk can be safely managed on cluded, 104 in each study phase; complete followup was obtained in 201 patients. GBS varied from 0 to 18 , with 15 (14\%) and 11 (11\%) patients having a GBS of 0 in the observational and interventional study phase, respectively. For patients with a GBS of 0 , hospital stay was shorter (6 versus 19 $\mathrm{h}, P<0.01$ ), and costs were lower (845 EUR versus 1272 EUR, $P=0.002$ ) in the interventional versus the observational study phase. For patients with a GBS $>0$, hospital stay duration did not significantly differ between study phases (189 versus $207 \mathrm{~h}, P=0.726$ ). No adverse event was observed in the patients sent home with a GBS of 0 during the interventional study phase.

Conclusions Implementing the GBS as a tool for triage of hospital outpatients who present with UGI bleeding allowed us to identify those who could safely be discharged for ambulatory management. Implementing this change in the hospital strategy significantly shortened hospital stay and decreased management costs.

an outpatient basis, allowing a reduced duration of hospital stay and decreased associated costs $[6,7]$. The Glasgow-Blatchford Bleeding score (GBS, Table 1) predicts the need for surgery, blood transfusion and any endoscopic hemostasis [8-11]. Its main advantage over other scores available for selecting patients at low risk of rebleeding is that it can be computed in a straightforward manner, without UGI endoscopy. A GBS of 0 has been shown to accurately predict a very low risk of requiring a clinical intervention, defined as blood transfusion, endoscopic hemostasis or surgical management for bleeding control [8].

We tested the hypothesis that, by implementing the GBS, selecting low-risk GI bleeders and safely managing them as outpatients, costs and duration of hospital stay can be reduced. 
Table 1 Glasgow-Blatchford score.

\begin{tabular}{|c|c|}
\hline & GBS \\
\hline \multicolumn{2}{|c|}{ Blood urea (mmol/l) } \\
\hline$<6.5$ & 0 \\
\hline $6.5-7.9$ & 2 \\
\hline $8-9.9$ & 3 \\
\hline $10-25$ & 4 \\
\hline$>25$ & 6 \\
\hline \multicolumn{2}{|c|}{ Hemoglobin: men (g/l) } \\
\hline$>129$ & 0 \\
\hline $120-129$ & 1 \\
\hline $100-119$ & 3 \\
\hline$<100$ & 6 \\
\hline \multicolumn{2}{|c|}{ Hemoglobin: women (g/l) } \\
\hline$>119$ & 0 \\
\hline $100-119$ & 1 \\
\hline$<100$ & 6 \\
\hline \multicolumn{2}{|c|}{ Systolic blood pressure $(\mathrm{mmHg})$} \\
\hline$>109$ & 0 \\
\hline $100-109$ & 1 \\
\hline $90-99$ & 2 \\
\hline$<90$ & 3 \\
\hline \multicolumn{2}{|l|}{ Other markers } \\
\hline $\mathrm{BPM} \geq 100$ & 1 \\
\hline Melena & 1 \\
\hline Syncope & 2 \\
\hline Liver disease* & 2 \\
\hline Heart failure* & 2 \\
\hline
\end{tabular}

Abbreviations: BPM, beats per minute; GBS, Glasgow-Blatchford Bleeding Score. Total score is obtained by adding the value of all items.

Adapted from Stanley A] et al [8].

* Based on past medical history, clinical or laboratory evidence.

\section{Patients and methods}

$\nabla$

Consecutive outpatients admitted to the Emergency Department of the University Hospital of Geneva (Geneva, Switzerland) with UGI bleeding were prospectively screened for inclusion in the study. Patients over 18 years of age with UGI bleeding defined as hematemesis or coffee ground emesis or with melena were eligible for inclusion. Exclusion criteria were pregnancy and hematochezia (to avoid all lower gastrointestinal bleeding, acknowledging that severe UGI bleeding may present with hematochezia). Vital signs, clinical history, physical examination and hemoglobin levels were obtained in the Hospital Emergency Department, at admission, to calculate the GBS. The study was divided into two consecutive phases:

- During the "observational" study phase (October 2009-August 2010), apart from a systematic calculation of the GBS, routine local clinical practice was unchanged: all patients received proton pump inhibitor (PPI) therapy and underwent an UGI endoscopy during the $12 \mathrm{~h}$ following hospital admission, under midazolam sedation or with orotracheal intubation for unstable patients. This was performed in the endoscopy unit or in the Emergency Department in the case of patient instability or when endoscopies were done during oncall hours. The physician responsible for the patient in the Emergency Department decided whether to discharge or to admit the patient without interference from the investigators.

- During the "interventional" study phase (January 2011 -January 2012), patients with a GBS of 0 were not admitted to the hospital and they received an appointment for an ambulatory UGI endoscopy during the following $48 \mathrm{~h}$. If the GBS was $>0$, patient management was identical to the observational study phase.

Vital parameters and hemoglobin levels were recorded and supportive measures such as drug administration, blood transfusion and resuscitation, were administered by the physician in charge (i.e., physician of the Emergency Department relayed by the physician in charge on the ward) and prospectively monitored. Endoscopic hemostasis during emergent UGI endoscopy was performed if deemed indicated by the endoscopist. All emergent endoscopies were reviewed daily by a panel of $\geq 2$ senior endoscopists. In the case of rebleeding, the decision to perform a surgical operation or not was taken by the patient and the medical team (physician in charge, endoscopist, surgeon and radiologist).

Follow-up was performed at 7 and 30 days by one of the investigators (MG) using hospital charts supplemented by face-to-face visits or phone calls. Data prospectively collected included vital signs, the presence of major comorbidities, results of blood analysis, endoscopic findings, PPI administration, number of red blood cell units transfused, rebleeding events, readmission, surgery and death. Hospital costs were calculated for patients with a GBS of 0 during each study phase. According to Swiss public healthcare tariffs, cost calculations included all real costs for the first $24 \mathrm{~h}$, calculated using the 2013 TARMED reimbursement rates plus, in the case of a hospital stay longer than $24 \mathrm{~h}$, a daily package of 686 EUR.

The study protocol was approved by the hospital ethics committee (Geneva University Hospital IRB) and it was registered in a publically accessible registry (clinicaltrials.gov \#NCT01029626); all patients gave written informed consent before inclusion in the study.

\section{Statistical analysis}

Categorical and continuous variables were described as percentages and by their median and range, respectively. Comparisons between groups were performed using Fisher's exact test for categorical data and the Mann-Whitney test for continuous variables. All $P$ tests were two-sided, and $P$ values $<0.05$ were considered to be statistically significant. All analyses were performed on an intention-to-treat basis including the 208 patients. Sample-size calculation was based on the assumption that duration of hospital stay for patients admitted with UGI bleeding would be shortened by $25 \%$ in the interventional study phase compared with the observational study phase. On the basis of 0.8 power, 104 patients were required in each study phase to detect a significant difference $(P=0.05)$. Analyses were performed using Prism version 6.00 for Windows (GraphPad Software, San Diego, CA, USA).

\section{Results}

\section{Patients}

Two-hundred and eight consecutive consenting patients with UGI bleeding were included in this study ( $\bullet$ Fig. 1 ). Nineteen patients were excluded in each study phase because they were unable $(n=12)$ or refused $(n=15)$ to give consent, or because blood urea level was not measured $(n=11)$. Follow-up at 7 and 30 days was obtained for $208(100 \%)$ and 198 (95\%) patients, respectively. At 30 days, 7 (3.4\%) patients had died and three (1.4\%) patients were lost to follow-up. 


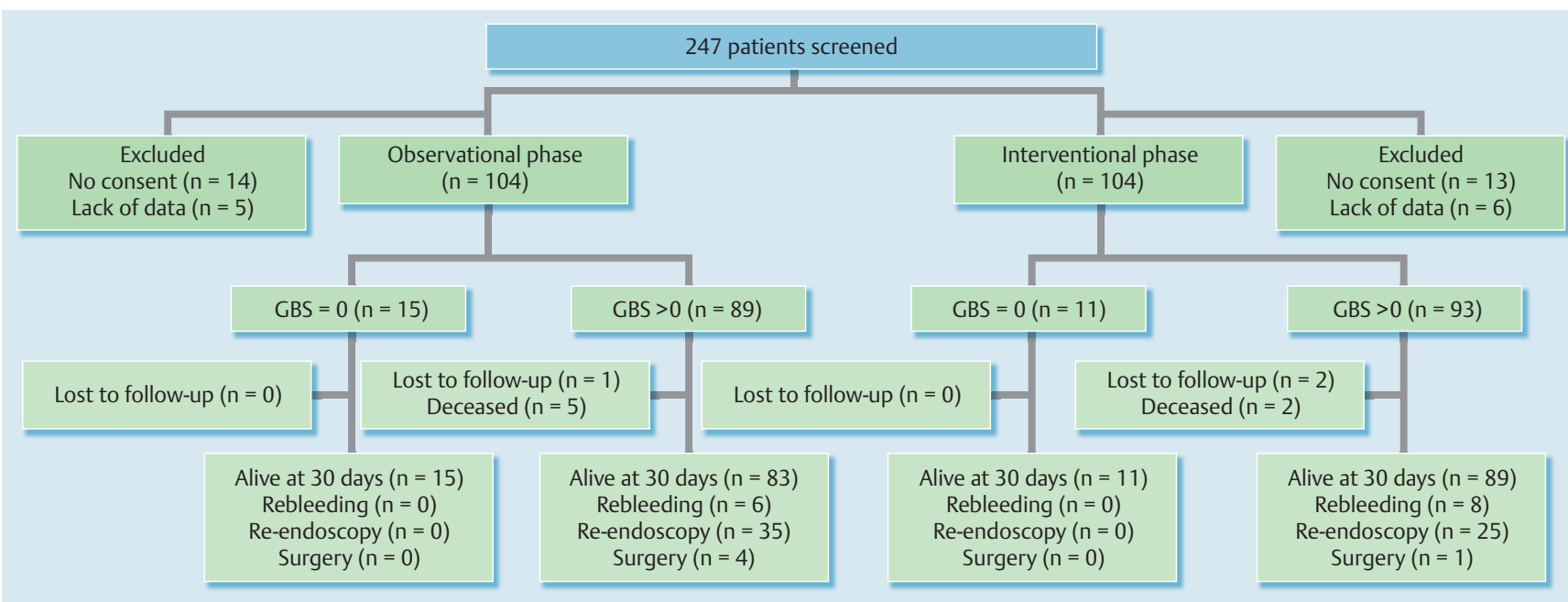

Fig. 1 Enrollment and outcome. Patients were enrolled in two consecutive phases: during the first, observational, phase, all patients had upper gastrointestinal endoscopy; during the second, interventional, phase, patients with a Glasgow-Blatchford Bleeding Score (GBS) of 0 were discharged with an appointment for upper gastrointestinal endoscopy during the following $48 \mathrm{~h}$.

Table 2 Patients' characteristics at baseline ${ }^{1}$

\begin{tabular}{|c|c|c|c|c|c|c|}
\hline & \multicolumn{3}{|c|}{$\begin{array}{l}\text { Observational study phase } \\
(n=104)\end{array}$} & \multicolumn{3}{|c|}{$\begin{array}{l}\text { Interventional study phase } \\
(n=104)\end{array}$} \\
\hline & $\begin{array}{l}G B S=0 \\
(n=15)\end{array}$ & $\begin{array}{l}G B S>0 \\
(n=89)\end{array}$ & $P$-value & $\begin{array}{l}G B S=0^{2} \\
(n=11)\end{array}$ & $\begin{array}{l}\text { GBS }>0^{2} \\
(n=93)\end{array}$ & $P$-value \\
\hline Age (years) & $35(21-91)$ & $69(20-99)$ & 0.0001 & $33(20-43)$ & $71(25-96)$ & 0.001 \\
\hline Men, n (\%) & $11(73)$ & $68(76)$ & NS & $10(91)$ & $58(62)$ & NS \\
\hline Urea (mmol/L) & $5(1.8-6.1)$ & $10(2-49.6)$ & 0.0001 & $4(2.6-5.8)$ & $11(2.4-38.2)$ & 0.001 \\
\hline $\mathrm{Hb}(\mathrm{g} / \mathrm{L})$ & $150(127-160)$ & $98(38-185)$ & 0.0001 & $150(136-174)$ & $86(46-156)$ & 0.001 \\
\hline $\mathrm{SBP}(\mathrm{mmHg})$ & $135(110-160)$ & $120(90-170)$ & NS & $130(110-145)$ & $120(70-170)$ & NS \\
\hline BPM & $75(61-93)$ & $96(61-150)$ & 0.048 & $69(53-99)$ & $89(59-150)$ & 0.001 \\
\hline $\mathrm{BPM}>100, \mathrm{n}(\%)$ & 0 & $35(39)$ & 0.001 & 0 & $31(33)$ & 0.030 \\
\hline Melena, n (\%) & 0 & $65(73)$ & 0.0001 & 0 & $71(76)$ & 0.0001 \\
\hline Syncope, n (\%) & 0 & $6(7)$ & NS & 0 & $11(12)$ & NS \\
\hline Liver disease, n (\%) & 0 & $33(37)$ & 0.049 & 0 & $24(26)$ & NS \\
\hline Heart failure, $n$ (\%) & 0 & $4(5)$ & NS & 0 & $3(3)$ & NS \\
\hline GBS & 0 & $9(1-17)$ & 0.0001 & 0 & $11(1-17)$ & 0.001 \\
\hline
\end{tabular}

Abbreviations: BPM, beats per minute; GBS, Glasgow-Blatchford Bleeding Score; NS, non-significant; SBP, systolic blood pressure.

1 Values are medians with ranges in parentheses except otherwise stated.

${ }^{2} P>0.05$ for all comparisons with the corresponding subgroup of patients in the observational study phase.

Patients' characteristics at baseline were similar for the two study phases $(\bullet$ Table 2$)$. As expected, compared with patients who presented with a GBS>0, those with a GBS of 0 were younger, had lower blood urea levels, higher hemoglobin levels and a lower heart rate during each study phase ( $\bullet$ Table 2). The distribution of GBS was similar for the two study phases, with $15(14 \%)$ and $11(11 \%)$ patients having a GBS of 0 during the observational and interventional study phase, respectively $(P=$ $0.530)(\odot$ Fig. 2).

\section{Endoscopic findings}

- Table 3 summarizes the endoscopic findings in each study phase. Endoscopic diagnoses in patients with a GBS of 0 were non-severe lesions, mostly Los Angeles grade A esophagitis, Forrest III duodenal ulcer or Mallory-Weiss tears. None of these patients had variceal bleeding. During the observational study phase, lesions listed as "other" in patients with a GBS of 0 consisted of gastric erosions $(n=1)$ and during the interventional study phase, they consisted of Mallory-Weiss tears $(n=5)$ and gastric erosions $(n=1)$.

\section{Outcomes, hospital stay and costs}

In both study phases, none of the patients with a GBS of 0 received any clinical intervention (apart from one patient who received a hemoclip [QuickClip, Olympus, Tokyo, Japan] for a small and superficial gastric erosion during the interventional study phase; this intervention was considered to be not needed by two senior endoscopists after reviewing the case on the following day). In contrast, $27 \%$ and $39 \%$ of patients with a GBS $>0$ received endoscopic hemostasis during the observational and interventional study phases, respectively. As expected, non-endoscopic outcomes (red blood cell transfusions and administration of PPI) were markedly different for patients with a GBS of 0 versus $>0$, in each study phase ( $\bullet$ Table 4 ). No significant difference in clinical outcomes was observed between study phases for patients with a GBS of 0 and for those with a GBS >0 ( $\bullet$ Table 4 ), apart 


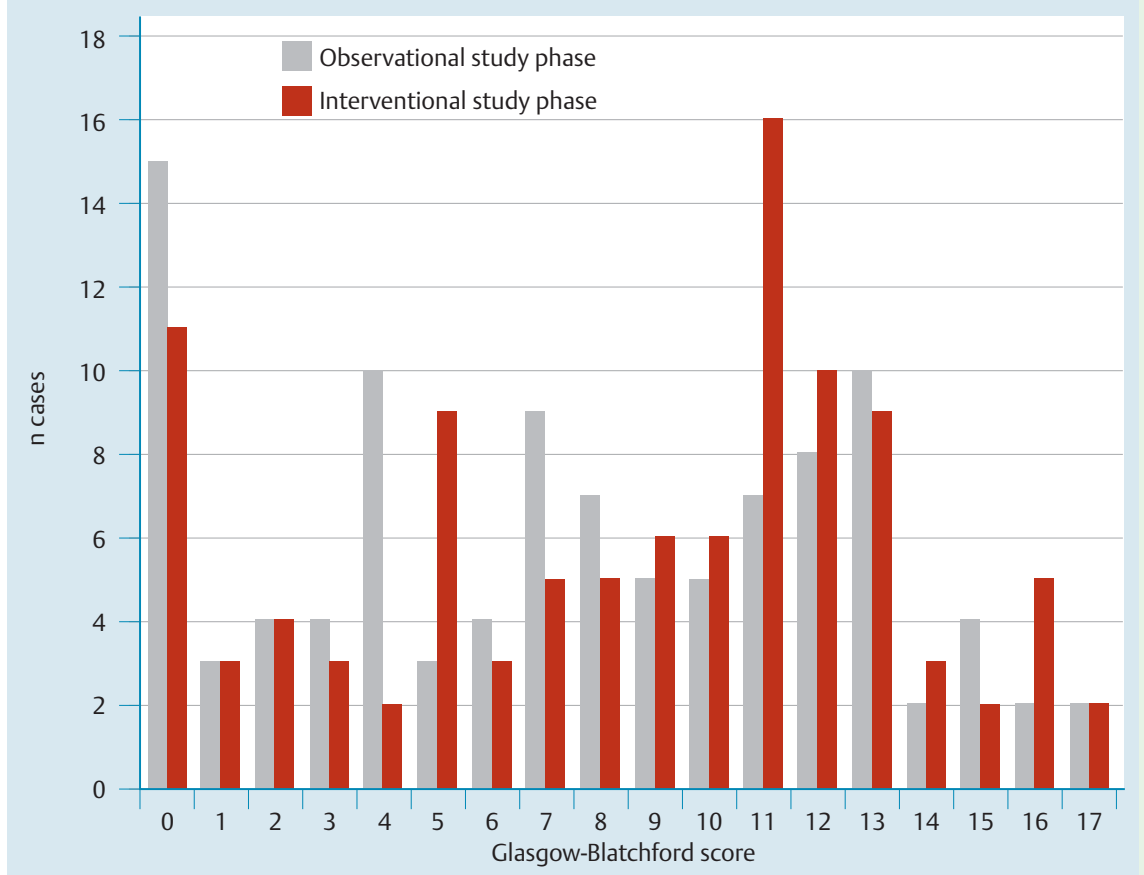

Fig. 2 Distribution of the number of patients per Glasgow-Blatchford Bleeding Score during the observational and interventional study phases.

Table 3 Endoscopic diagnosis'.

\begin{tabular}{|c|c|c|c|c|c|c|}
\hline \multirow[b]{2}{*}{ Diagnosis } & \multicolumn{3}{|c|}{$\begin{array}{l}\text { Observational study phase } \\
(n=104)\end{array}$} & \multicolumn{3}{|c|}{$\begin{array}{l}\text { Interventional study phase } \\
(n=104)\end{array}$} \\
\hline & $\begin{array}{l}G B S=0 \\
(n=15)\end{array}$ & $\begin{array}{l}\text { GBS }>0 \\
(n=89)\end{array}$ & $P$-value & $\begin{array}{l}G B S=0 \\
(n=11)\end{array}$ & $\begin{array}{l}G B S>0 \\
(n=93)\end{array}$ & $P$-value \\
\hline No lesions & $8(53)$ & $10(11)$ & 0.0005 & $3(27)$ & $10(11)$ & NS \\
\hline Blood in upper digestive tract & $1(7)$ & $25(28)$ & NS & 0 & $26(28)$ & NS \\
\hline EV or GV & 0 & $21(24)$ & 0.037 & 0 & $16(17)$ & NS \\
\hline Reflux esophagitis & $6(40)$ & $22(25)$ & NS & $1(9)$ & $28(30)$ & NS \\
\hline PHT gastropathy & 0 & $19(21)$ & NS & 0 & $6(6)$ & NS \\
\hline Gastric ulcer & 0 & $22(25)$ & 0.036 & 0 & $33(35)$ & 0.015 \\
\hline Duodenal ulcer & 0 & $22(25)$ & 0.036 & $1(9)$ & $24(26)$ & NS \\
\hline Other lesion ${ }^{2}$ & $1(7)$ & $10(11)$ & NS & $6(54)$ & $9(10)$ & 0.001 \\
\hline
\end{tabular}

Abbreviations: EV, esophageal varices; GBS, Glasgow-Blatchford Bleeding Score; GV, gastric varices; NS, non-significant; PHT, portal hypertension.

${ }^{1}$ Values are numbers of patients with percentages in parentheses; values may add to more than $100 \%$ as some patients had several diagnoses.

2 Mallory-Weiss tears, gastrointestinal stromal tumor, gastric arterio-venous ectasia, angiodysplasia, Dieulafoy's lesion.

Table 4 Clinical outcome'.

\begin{tabular}{|c|c|c|c|c|c|c|}
\hline & \multicolumn{3}{|c|}{$\begin{array}{l}\text { Observational study phase } \\
(n=104)\end{array}$} & \multicolumn{3}{|c|}{$\begin{array}{l}\text { Interventional study phase } \\
(n=104)\end{array}$} \\
\hline & $\begin{array}{l}G B S=0 \\
(n=15)\end{array}$ & $\begin{array}{l}\text { GBS >0 } \\
(n=89)\end{array}$ & $P$-value & $\begin{array}{l}G B S=0 \\
(n=11)\end{array}$ & $\begin{array}{l}G B S>0 \\
(n=93)\end{array}$ & $P$-value \\
\hline $\begin{array}{l}\text { Clinical intervention (transfusion, } \\
\text { surgery or hemostasis), } \mathrm{n}(\%)\end{array}$ & 0 & $57(64)$ & 0.0001 & $1(9)$ & $63(68)$ & 0.0001 \\
\hline Hemostasis, n (\%) & 0 & $24(27)$ & 0.019 & $1(9)$ & $36(39)$ & NS \\
\hline Surgery, n (\%) & 0 & $2(2)$ & NS & 0 & $1(1)$ & NS \\
\hline Death at 30 days, $n(\%)$ & 0 & $5(6)$ & NS & 0 & $2(2)$ & NS \\
\hline Blood transfusion, $\mathrm{n}(\%)$ & 0 & $54(61)$ & 0.0001 & 0 & $59(63)$ & 0.0001 \\
\hline PPI duration (days) & $1(0-2)$ & $3(0-6)$ & 0.0004 & 0 & $3(0-3)$ & 0.001 \\
\hline Hospital stay duration (h) & $19(5-148)$ & $189(5-816)$ & 0.0001 & $6(1-13)^{2}$ & $207(7-1035)$ & 0.0001 \\
\hline
\end{tabular}

Abbreviations: GBS, Glasgow-Blatchford Bleeding Score; NS, non-significant; PPI, proton pump inhibitors.

1 Values are medians with ranges in parentheses except otherwise stated.

2 $P=0.010$ for comparison with the observational study phase; all other comparisons with the observational study phase showed no statistical significance. 


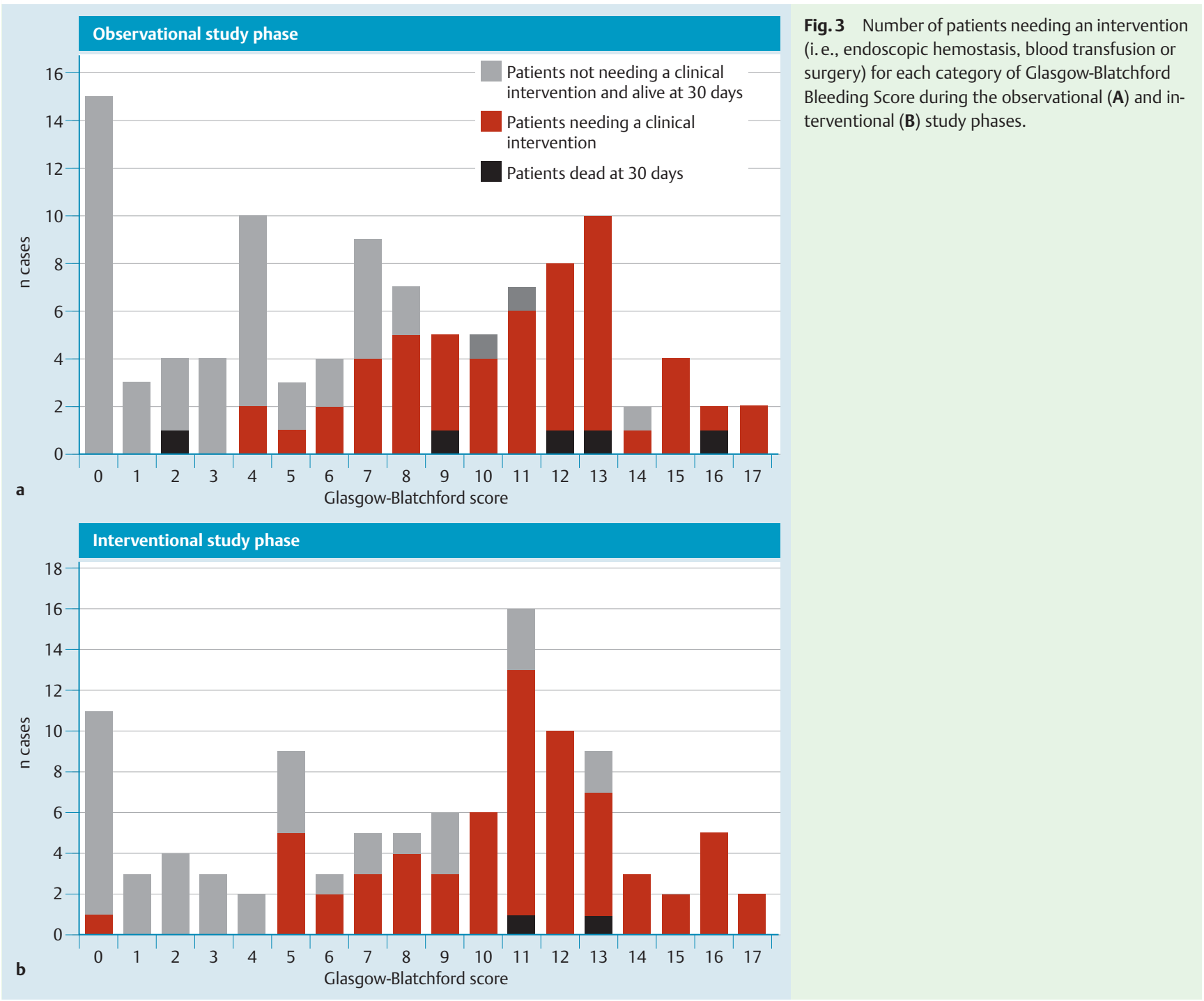

from the duration of hospital stay of patients with a GBS of 0: this decreased by $68 \%$ during the interventional study phase, from 19 $\mathrm{h}(5-148 \mathrm{~h})$ to $6 \mathrm{~h}(1-13 \mathrm{~h})(P=0.010)$. Concomitantly, in patients with a GBS of 0 , median hospitalization costs decreased by $34 \%$, from 1272 EUR (553 - 4296 EUR) to 845 EUR (336 - 1441 EUR ) $(P$ $=0.002)$. None of the patients in the group with a GBS of 0 were readmitted, presented rebleeding or required a repeat endoscopy. All patients with a GBS of 0 were alive at the 30-day follow-up ( $\bullet$ Fig.3).

\section{Discussion \\ $\nabla$}

This study was aimed at defining whether applying the GBS score to patients presenting at an Emergency Department with UGI bleeding may alter the hospital stay and costs by selecting patients who will or will not need an intervention (surgery, blood transfusion or endoscopic hemostasis). We found that duration of hospital stay and costs were drastically reduced in low-risk UGI bleeders by implementing a policy of outpatient management.

All of our patients with a GBS of 0 were alive at 30 days without having presented rebleeding or requiring repeat endoscopy. This is in line with the large multicentric study by Stanley et al. which showed that, among a total of 228 patients who presented with a GBS of 0 , none had presented rebleeding or had needed repeat endoscopy during follow-up [8]. These authors also reported that the introduction of the GBS into their clinical practice was associated with a reduced proportion of low-risk patients admitted to the hospital, down to $32 \%$ (a formal protocol to discharge patients with a GBS of 0 was not used in that study).

We selected a risk score that can be computed before endoscopy to maximize benefits in terms of hospital costs and capacity overload in the Emergency Department. Among three risk scores available for assessing the severity of UGI bleeding before performing endoscopy, we selected the GBS because several studies have demonstrated its superiority over the Rockall score in predicting the need for clinical intervention, and the GBS is simpler to calculate than the "artificial neural network" [12]. The GBS is promptly and easily calculated, allowing an immediate decision to perform UGI endoscopy or to discharge the patient as soon as hemoglobin and urea blood levels are available. We experienced difficulties in implementing a systematic measurement of urea blood level in the Emergency Department of our hospital, which partly accounts for the relatively long duration of hospital stay observed in some of our patients who had a GBS of 0. This may also be the case in other hospitals as Stanley et al. did not obtain urea blood levels for $4 \%$ of their patients (similar to the $4.4 \%$ ob- 
served in our screened patients) [8]. Nevertheless, based on the results of the present study, the GBS has now been formally implemented in our hospital algorithm for the management of outpatients who present with UGI bleeding.

To the best of our knowledge, all other studies that used preendoscopy risk scores to select low-risk patients for a non-admission policy were observational:

- In a retrospective audit of two hospitals, Robins et al. showed that discharging selected UGI bleeders without endoscopy was safe: severity of UGI bleeding was calculated based on the GBS supplemented with clinical parameters; $9 \%$ of patients had UGI endoscopy in one hospital and their outcome was similar to that of patients who had presented to another hospital where endoscopy was performed more liberally (in $74 \%$ of patients) [13]

- In a mostly retrospective analysis, the policy of not admitting low-risk patients and scheduling UGI endoscopy on the next working day was evaluated in 139 patients over a 5 -year period. It was found to be safe as none of the patients received endoscopic therapy, blood transfusion or surgery for UGI bleeding. A GBS $\leq 2$ supplemented with clinical criteria was used to select low-risk bleeders [14].

One limitation of our study is the relatively small number of patients with a GBS of 0 who were included, particularly in the second study phase $(\mathrm{n}=11)$. However, this proportion $(12.5 \%)$ lies in the range of previous studies (5-22\%) [12]. Although the population of patients with a GBS of 0 was small, the reduction in duration of hospital stay and decreased costs were highly significant, which were in line with our basic hypothesis. Another limitation is that our study is a single center and single country study. Despite that, studies performed in other countries, using a GBS of 0 as cut-off predicted a good and safe clinical evolution. Similar results should be expected in reproducing this study elsewhere. In an observational study, Le Jeune et al. have reported that patients with a GBS $\leq 2$ could also be considered for early discharge, potentially doubling the number of patients eligible for a "non-admission" management [15]. In our study, $3(5 \%)$ of our 59 patients with a GBS $\leq 4$ needed an intervention and one patient with a GBS of 2 died at day 2 post-gastrectomy for a gastric cancer discovered at UGI endoscopy performed upon admission to our hospital. On the other hand, the strengths of our study include its prospective, interventional, design with the inclusion of a high number of patients and a 30-day follow-up rate of $95 \%$.

We included prospectively all patients with UGI bleeding regardless of whether the bleeding was variceal or not. It was important not to exclude cirrhotic patients because these patients often present with non-variceal bleeding. As in other trials [8], the GBS showed its ability to discriminate severe from non-severe variceal bleeding as well as non-variceal bleeding.
In conclusion, we showed that implementing a triage policy based on the GBS for selecting out-of-hospital UGI bleeders who are eligible for outpatient management effectively reduces duration of hospital stay and costs.

\section{Competing interests: None.}

\section{References}

1 Gilbert DA. Epidemiology of upper gastrointestinal bleeding. Gastrointest Endosc 1990; 36: 8-13

2 Laine L, Yang H, Chang SC et al. Trends for incidence of hospitalization and death due to GI complications in the United States from 2001 to 2009. Am J Gastroenterol 2012; 107: 1190 - 1195 quiz 1196

3 British Society of Gastroenterology Endoscopy Committee. Non-variceal upper gastrointestinal haemorrhage: guidelines. Gut 2002; 51: iv1-iv6

4 Viviane A, Alan BN. Estimates of costs of hospital stay for variceal and nonvariceal upper gastrointestinal bleeding in the United States. Value Health 2008; 11: 1-3

5 Cryer BL, Wilcox CM, Henk HJ et al. The economics of upper gastrointestinal bleeding in a US managed-care setting: a retrospective, claimsbased analysis. J Media Econ 2010; 13: 70-77

6 Longstreth GF, Feitelberg SP. Successful outpatient management of acute upper gastrointestinal hemorrhage: use of practice guidelines in a large patient series. Gastrointest Endosc 1998; 47: 219-222

7 Cipolletta L, Bianco MA, Rotondano G et al. Outpatient management for low-risk nonvariceal upper GI bleeding: a randomized controlled trial. Gastrointest Endosc 2002; 55: 1 -5

8 Stanley AJ, Ashley D, Dalton HR et al. Outpatient management of patients with low-risk upper-gastrointestinal haemorrhage: multicentre validation and prospective evaluation. Lancet 2009; 373: 42-47

9 Blatchford O, Murray WR, Blatchford M. A risk score to predict need for treatment for upper-gastrointestinal haemorrhage. Lancet 2000; 356: $1318-1321$

10 Dicu D, Pop F, Ionescu D et al. Comparison of risk scoring systems in predicting clinical outcome at upper gastrointestinal bleeding patients in an emergency unit. Am J Emerg Med 2013; 31: 94-99

11 Laursen SB, Hansen JM, Schaffalitzky de Muckadell OB. The Glasgow Blatchford score is the most accurate assessment of patients with upper gastrointestinal hemorrhage. Clin Gastroenterol Hepatol 2012; 10: 1130-1135, e1

12 Stanley AJ. Update on risk scoring systems for patients with upper gastrointestinal haemorrhage. World J Gastroenterol 2012; 18: 27392744

13 Robins GG, Sarwar MS, Armstrong MJ et al. Evaluation of the need for endoscopy to identify low-risk patients presenting with an acute upper gastrointestinal bleed suitable for early discharge. Postgrad Med J 2007; 83: $768-772$

14 McLaughlin C, Vine L, Chapman L et al. The management of low-risk primary upper gastrointestinal haemorrhage in the community: a 5year observational study. Eur J Gastroenterol Hepatol 2012; 24: 288 293

15 Le JeuneIR, Gordon AL, Farrugia D et al. Safe discharge of patients with low-risk upper gastrointestinal bleeding (UGIB): can the use of Glasgow-Blatchford Bleeding Score be extended? Acute Med 2011; 10: $176-181$ 